\title{
Biomechanical and energetic effects of a stance-control orthotic knee joint
}

\author{
Angelika Zissimopoulos, MS; ${ }^{1-2 *}$ Stefania Fatone, PhD, BPO (Hons); ${ }^{1,3}$ Steven A. Gard, PhD ${ }^{1-3}$ \\ ${ }^{1}$ Northwestern University Prosthetics Research Laboratory and Rehabilitation Engineering Research Program, \\ Department of Physical Medicine and Rehabilitation, Feinberg School of Medicine, Northwestern University, Chicago, \\ $I L ;{ }^{2}$ Department of Biomedical Engineering, Robert R. McCormick School of Engineering and Applied Science, \\ Northwestern University, Evanston, IL; ${ }^{3}$ Jesse Brown Department of Veterans Affairs Medical Center, Chicago, IL
}

\begin{abstract}
Users of traditional knee-ankle-foot orthoses (KAFOs) walk with either locked or unlocked knee joints depending on the level of stability required. Some users may benefit from new stance-control KAFOs that prevent stancephase knee flexion but allow swing-phase flexion. We collected data from nine nondisabled adults who walked with KAFOs that incorporated the Horton Stance-Control Orthotic Knee Joint (SCOKJ) in the locked, unlocked, and auto (which provides knee stability during stance phase and knee flexion during swing phase) modes to investigate the biomechanical and energetic effects of stance-control orthoses. Studying nondisabled subjects allowed us to analyze the effects of stancecontrol orthoses in a homogenous population. In general, gait kinematics for the auto and unlocked modes were more similar than for the auto and locked modes. Despite the elimination of hip hiking in the auto mode, oxygen cost was not different between the auto and locked modes $(p>0.99)$. The SCOKJ allowed our nondisabled subjects to walk with a more normal gait pattern; however, future research should explore the effect of stance-control orthoses on persons with gait pathology.
\end{abstract}

Key words: energy expenditure, gait, KAFO, kinematics, knee-ankle-foot orthosis, orthotic device, oxygen cost, rehabilitation, SCOKJ, stance-control.

\section{INTRODUCTION}

Knee-ankle-foot orthoses (KAFOs) are often prescribed for patients with weakness of the muscles that control the knee and/or loss of structural integrity of the knee joint. Traditionally, the knee joints used in these orthoses were either locked or unlocked during ambulation depending on the level of stability required. However, walking with a locked knee for the entire gait cycle requires specific gait compensations, including hip hiking, vaulting, and circumduction, to create sufficient toe clearance during swing phase [1-3]. Other studies have demonstrated that these gait compensations tend to increase a person's energy expenditure [4-8]. While some patients may require the stability of a locked knee for the entire gait cycle, a subset of patients would benefit from a device that locks during stance phase to provide support for the weight-bearing limb and unlocks during swing phase to allow knee flexion, providing sufficient toe clearance by shortening the leg. Components exhibiting this behavior are referred to as "stance-control" devices.

Within the past few years, a number of stance-control orthoses have been developed for use in KAFOs. Each of

\footnotetext{
Abbreviations: ANOVA $=$ analysis of variance, $\mathrm{KAFO}=$ knee-ankle-foot orthosis, MTP = metatarsal phalangeal, $\mathrm{PCI}=$ physiological cost index, $\mathrm{PP}=$ peak-to-peak, SCOKJ = StanceControl Orthotic Knee Joint, SD = standard deviation, VA = Department of Veterans Affairs, VACMARL = VA Chicago Motion Analysis Research Laboratory.

*Address all correspondence to Angelika Zissimopoulos, MS; Northwestern University Prosthetics Research Laboratory and Rehabilitation Engineering Research Program, 345 E Superior St, RIC 1441, Chicago, IL 60611; 312-238-6500; fax: 312-238-6510. Email: kikiz@northwestern.edu DOI: 10.1682/JRRD.2006.09.0124
} 
these joints uses a different design strategy, but all prevent stance-phase knee flexion to provide stability, while allowing swing-phase knee flexion. Currently, limited research has been conducted on KAFOs in general [9] and in particular on the effects that stance-control KAFOs may have on a user's gait. To date, six studies have evaluated stance-control KAFOs [8,10-14], with only two of these investigating commercially available joints: McMillan et al. fitted three KAFO users with Horton's weightactivated Stance-Control Orthotic Knee Joint (SCOKJ ${ }^{\circledR}$ ) (Horton Technology, Inc, Little Rock, Arkansas) [13] and Hebert and Liggins fitted a single postpolio subject with the ankle-motion activated SCOKJ [10]. In general, these studies indicated that kinematic parameters, especially knee flexion during swing phase and pelvic motion, improved when subjects walked with a stance-control device compared with a completely locked knee joint.

The largest study evaluating stance-control KAFOs was conducted by Irby et al. [11]. Thirteen experienced KAFO users and eight novice users were tested while walking with an experimental stance-control KAFO in both the locked-knee and stance-control configurations. As expected, both groups achieved significantly increased knee flexion during swing phase in the stancecontrol configuration. Additionally, vaulting was reduced for both groups. However, only the novice users exhibited significantly increased speed, cadence, and singlelimb support time and significantly reduced pelvic obliquity, which indicates a reduction in hip hiking. These results suggested that, unlike the novice KAFO users, the experienced users' deeply ingrained walking habits did not allow them to fully benefit from the increased knee flexion during swing phase.

Lastly, four of these studies, all with less than five subjects, assessed energy expenditure while subjects walked with a stance-control KAFO [8,10,12-13]. Overall, the results were not consistent; some subjects exhibited lower oxygen cost, oxygen consumption, physiological cost index (PCI), or heart rate in the stance-control setting compared with the locked setting, while others did not. Due to the small sample sizes, the statistical significance for the energy expenditure data could not be determined.

This study investigated the biomechanical and energetic effects of a stance-control orthosis on the gait of nondisabled persons. This study was conducted using stancecontrol knee joints that allowed three modes of operation: unlocked (which allowed knee flexion throughout the gait cycle), locked (which prevented knee flexion throughout the gait cycle), and automatic (auto) (which blocked knee flexion during stance phase and allowed knee flexion during swing phase). We hypothesized that walking with the knee joints in the auto mode would produce gait characteristics more closely resembling those obtained with the knee joints unlocked than with the knee joints locked. We further hypothesized that allowing knee flexion during swing phase through the use of a stance-control orthosis would yield lower energy expenditure compared with locked-knee gait.

\section{METHODS}

Nine nondisabled subjects (five females and four males) were enrolled in the study. All subjects had no known musculoskeletal or neuromuscular problems. This study was approved by the Northwestern University Institutional Review Board, and all subjects provided informed consent prior to participation. Subject-specific data were collected, including age, height, mass (while wearing the orthosis), and foot length (while wearing shoes). Subjects had a mean \pm standard deviation (SD) age, height, and mass of $25 \pm 2 \mathrm{yr}, 173 \pm 8 \mathrm{~cm}$, and $69 \pm$ $10 \mathrm{~kg}$, respectively.

For this study, the Horton SCOKJ was chosen as a representative stance-control device for examining the biomechanical and energetic effects of stance-control orthoses on gait. As mentioned previously, the SCOKJ allows three modes of operation: unlocked, locked, and auto (stancecontrol). When users walk with this knee joint in the unlocked mode, it is free to flex during both stance and swing phase, while in the locked mode it remains locked throughout the gait cycle. When the knee joint is in the auto mode, it blocks flexion during stance phase to prevent the knee from collapsing, but allows extension. During swing phase, knee motion is unrestricted. The SCOKJ can be fabricated so that it is activated either by weight-bearing or ankle motion. Ankle-motion activation was used in this study, with ankle plantar flexion and dorsiflexion engaging and disengaging the knee joint, respectively.

The study consisted of three stages: fabrication, dynamic adjustment and training, and data collection. A qualified orthotist unilaterally cast each subject (left or right side determined by random assignment) and a stance-control KAFO was custom fabricated. The orthoses were composed of polypropylene thigh, calf, and foot sections with carbon-fiber reinforcement for additional 
rigidity. The calf and foot sections were articulated with double-action ankle joints and the thigh section was attached with the SCOKJ.

After subjects were fitted unilaterally with the stancecontrol KAFO, they were trained to walk with the device both on level ground and on a Cosmed model T170 treadmill (Cosmed, Rome, Italy) for 20-to-30 minutes each day, for up to 10 days or until they could reliably engage and disengage the knee joint. Subjects needed an average of 7 days to become comfortable operating the device. Instruction and supervision were provided during all training sessions. Subjects were taught how to disengage the knee joint, a process that required them to create a small knee-extension movement in late stance phase which, when coupled with dorsiflexion of the ankle (assisted by plantar flexion resistance from the double-action ankle joints), allowed the cam to disengage and permitted the knee to flex during swing phase. Following training, subjects participated in a two-part data collection session that consisted of a gait evaluation (overground walking) and an energy expenditure evaluation (treadmill walking).

All data were collected at the Department of Veterans Affairs (VA) Chicago Motion Analysis Research Laboratory (VACMARL) of the Jesse Brown VA Medical Center (Chicago, Illinois). During the gait evaluation, bilateral kinematic data were acquired with eight Eagle Digital RealTime cameras (Motion Analysis Corporation, Santa Rosa, California) sampled at $120 \mathrm{~Hz}$. The bilateral gait evaluation was performed with the Helen Hayes marker set [15]. Retroreflective markers were applied directly to the skin (on the nonorthotic side) or orthosis (on the orthotic side) with double-sided hypoallergenic tape. Specifically, on the nonorthotic side, markers were placed on the shoe at the dorsum of the foot over the metatarsal phalangeal (MTP) joint (approximately over the third ray), on the lateral malleolus, on the anterior surfaces of the shank and thigh segments, and on the lateral femoral epicondyle. On the orthotic side, markers were placed on the shoe on the dorsum of the foot over the MTP joint (approximately over the third ray), on the lateral mechanical ankle joint axis of rotation, on the anterior surface of the calf and distal thigh Velcro ${ }^{\circledR}$ straps, and on the mechanical knee joint over the axis of rotation. Markers were also placed on the pelvis over each anterior superior iliac spine and on the midpoint between the posterior superior iliac spines. The positions of four additional markers, placed on the medial malleolus and the medial epicondyle on the nonorthotic side and on the medial mechanical ankle and knee joint axes of rotation on the
KAFO, were recorded during a standing static trial to define the knee and ankle joint centers and axes (Figure 1). These additional markers were removed before data collection from the walking trials.

The bilateral gait evaluation was performed with a total of five testing conditions. At least three walking trials were collected for each condition. For the first three conditions, subjects were asked to walk at their freely selected speed, with the orthosis operating in each of the three modes. The testing order for the three freely selected speed conditions was randomized for each subject to

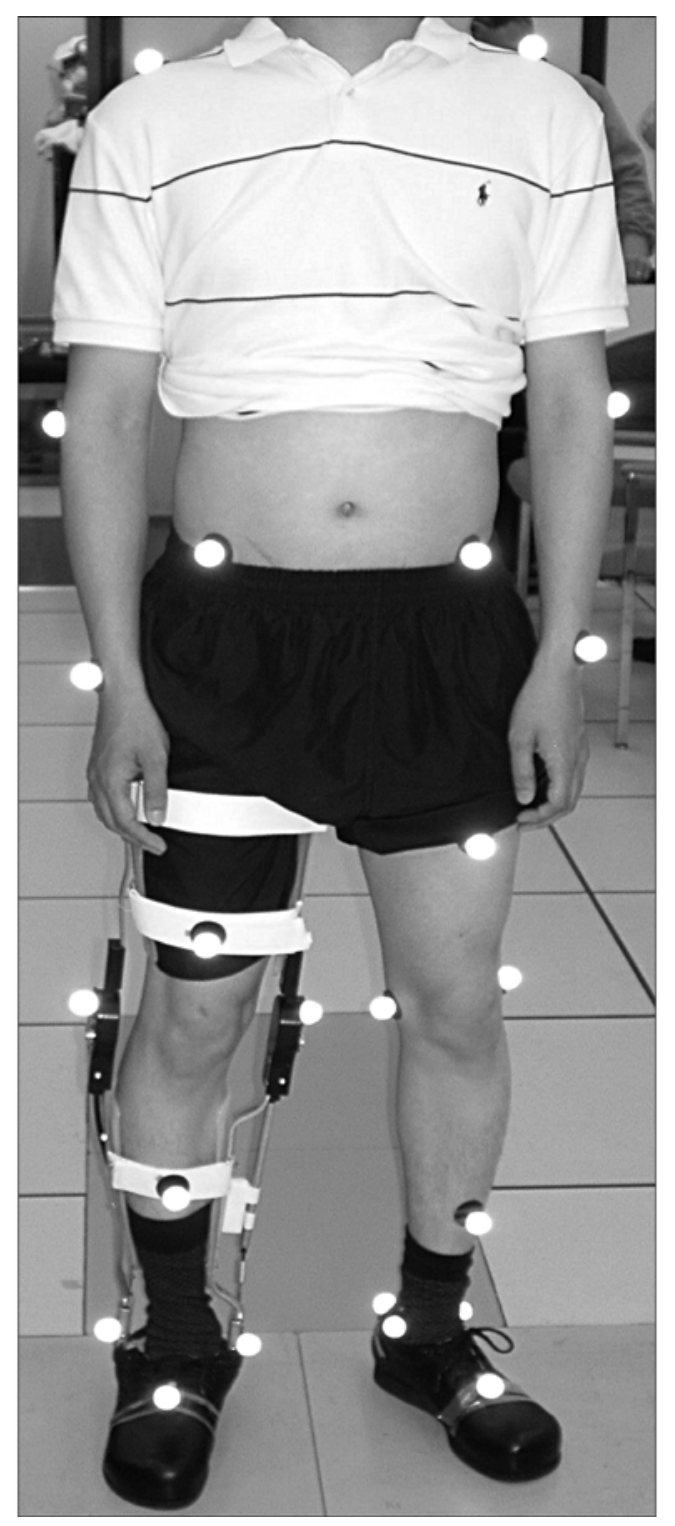

Figure 1.

Helen Hayes marker setup. 
reduce the potential effects of fatigue. The time required for the subject to cross the data collection volume (of a known distance) was recorded with a stopwatch for each trial and then averaged. The longest average walking time (slowest speed) from among the three modes was noted. To create a speed-matched data set, we had subjects walk in the other two modes at the same speed as the slowest mode. Subjects were timed to ensure that they consistently walked at this slow speed. For example, each subject walked at their freely selected speed in the unlocked, locked, and auto modes. If the slowest walking speed corresponded to the locked mode, the subject would be retested in the auto and unlocked modes at this slowest speed, creating a total of five walking conditions.

For the energy expenditure evaluation, data were collected with a Cosmed K4b2 portable spirometer and a Cosmed model T170 treadmill. Subjects were required to fast for 2 hours prior to the start of this portion of the experiment to eliminate the effect of food metabolism on energy expenditure measurements. Once the ambient conditions were recorded (ambient temperature and humidity), an oronasal mask was fitted to the subject. Adequate fit of the mask was evaluated as minimal leakage of air from the edges of the mask when a hand was placed over the turbine aperture while the subject was exhaling. Energy expenditure data were collected for the same five conditions, in the same order, and at the same walking speed calculated for each condition in the gait evaluation. However, only the three speed-matched conditions were used for the analysis of energy expenditure data. The energy expenditure test consisted of three stages: (1) 5 minutes of preexercise resting energy expenditure, which began once calibration was completed and the subject was fitted with the Cosmed equipment; (2) 7 minutes of walking energy expenditure on the treadmill for each of the five walking conditions (for a total of 35 minutes); and (3) at least 5 minutes of postexercise resting energy expenditure in a comfortable seated position or until heart rate returned to preexercise levels. To minimize fluctuations in data, we kept the laboratory quiet and allowed no interruptions during the energy expenditure sessions.

\section{DATA ANALYSIS}

Gait analysis data were processed with OrthoTrak (Motion Analysis Corporation). We used MATLAB (The MathWorks Inc, Natick, Massachusetts) and Excel (Microsoft Corp, Redmond, Washington) to generate kinematic graphs and calculate temporospatial data. Additionally, for comparison purposes, we chose 10 control subjects from our database, which contains data from nondisabled subjects walking at their freely selected very slow, slow, normal, fast, and very fast walking speeds. Control subjects were chosen so that their average age and very slow or slow walking speeds were approximately the same as those of the nine subjects in this study. The mean and SD for these data are plotted as shaded regions on the figures presented throughout this article.

Energy expenditure data were processed with the Cosmed software. For each of the 7-minute walking conditions, data collected during the first and last minutes were discarded. The average rate of oxygen consumption (milliliters of oxygen per kilogram per minute) for each condition was obtained by averaging the middle 5 minutes of oxygen consumption data for that condition. Average oxygen cost (milliliters of oxygen per kilogram per meter) was obtained by dividing the average oxygen consumption rate by walking speed (in meters per minute). Finally, using the unlocked mode as a baseline, we converted the locked- and auto-mode values into a percent increase relative to the unlocked mode.

Statistical analyses were conducted using SPSS software (SPSS Inc, Chicago, Illinois). If normality and sphericity were met, the three main effects (unlocked, locked, and auto modes) were tested at a significance level of $\alpha=$ 0.05 using a repeated-measures analysis of variance (ANOVA) with a Bonferroni correction factor (automatically applied by the software). If the sphericity condition was not met, the Greenhouse-Geisser correction was used. For data sets that were not normally distributed, the nonparametric Freidman two-way ANOVA with a significance level set at $\alpha=0.05$ was first applied to determine if any significant differences were present among the three modes. If significant differences were present, the Wilcoxon signed rank test with a Bonferroni correction was used to detect which of the pairwise comparisons were significant. Since three comparisons were made, the significance level for the Wilcoxon signed rank test was set at $\alpha=0.016$ [16].

When it was useful to compare orthotic- and nonorthotic-side data for a particular variable (normally distributed data sets), we performed a paired $t$-test with a Bonferroni correction on the unlocked, locked, and auto modes separately. For data sets that were not normally distributed, a Wilcoxon signed rank test with a Bonferroni correction was performed [16]. In both cases, the Bonferroni 
correction was not applied automatically by the software, and therefore, significance was manually set at $\alpha=0.016$.

\section{RESULTS}

\section{Temporospatial Data}

Data from the freely selected walking speed trials indicated that subjects walked significantly faster and with a higher cadence in the unlocked mode compared with the locked mode ( $p=0.01$ and $p=0.005$, respectively) but not significantly faster or with a higher cadence compared with the auto mode ( $p=0.09$ and $p=$ 0.22 , respectively). Speed and cadence for the locked and auto modes were not significantly different $(p>0.99)$ (Figure 2). Data from the speed-matched walking trials are summarized in the Table.

(a)

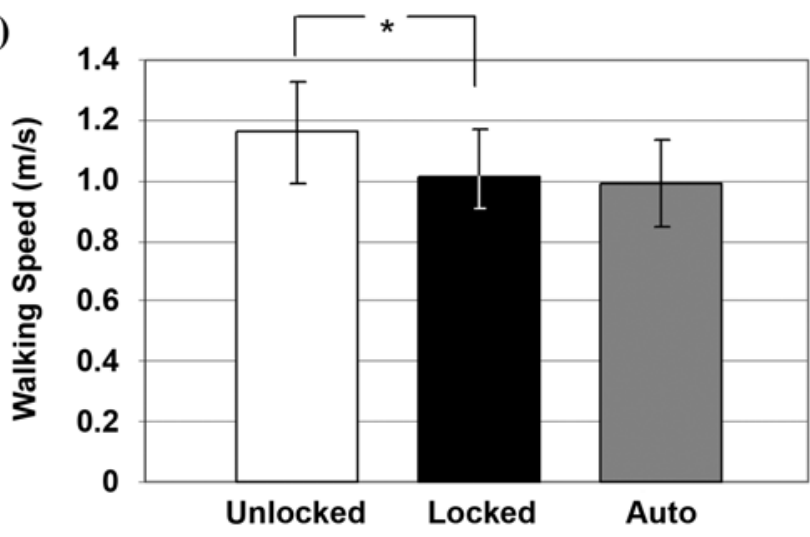

(b)

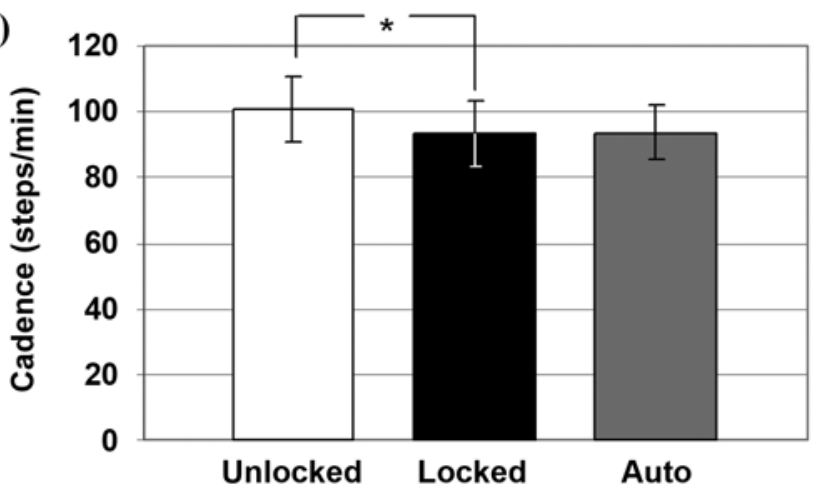

Figure 2.

Average (a) freely selected walking speed and (b) cadence shown with 1 standard deviation about mean for all three modes. * indicates statistically significant difference.

\section{Kinematics}

Subjects demonstrated a significant ankle dorsiflexion bias in all three modes on the orthotic side compared with the nonorthotic side $(p<0.001)$ (Figure 3).

Orthotic-side locked-mode knee range of motion was reduced compared with the unlocked and auto modes. On the orthotic side, peak-to-peak (PP) stance-phase knee flexion for the locked mode was significantly lower than for the auto $(p=0.009)$ and unlocked modes $(p<0.001)$ (Figure 4). Additionally, PP stance-phase knee flexion was significantly reduced in the auto mode when compared with the unlocked mode $(p<0.001)$. PP locked-mode knee flexion during swing phase was significantly lower than PP knee flexion during swing phase in the unlocked ( $p<$ $0.001)$ and auto modes $(p<0.001)$. However, no difference was noted between the auto- and unlocked-mode PP knee flexion during swing phase $(p=0.9)$.

On average, orthotic-side swing-phase PP pelvic obliquity (which is used to identify hip hiking) was significantly greater when subjects walked in the locked mode compared with the unlocked $(p=0.003)$ and auto modes ( $p=0.01$ ) (Figure 5) but was not significantly different between the unlocked and auto modes $(p=0.6)$.

The projection of the thigh angle in the coronal plane depicted thigh motion toward or away from the body and was used as an approximation of circumduction (definition adapted from Kerrigan et al. [2]). Subjects 5, 6, and 9 exhibited an increased thigh angle during swing phase in the locked mode as shown in Figure 6.

Although substantial variability in sagittal plane pelvic motion was found among subjects, one consistent result was that all subjects exhibited significantly increased PP pelvic tilt during orthotic-side swing phase in the locked mode compared with the unlocked ( $p<$ $0.001)$ and auto modes $(p=0.005)$ (Figure 7). PP pelvic tilt for the unlocked and auto modes was not significantly different during swing phase on the orthotic side $(p=0.3)$.

\section{Energy Expenditure}

Speed-matched data were used to assess energy expenditure. Average unlocked-mode oxygen cost was significantly lower than average oxygen cost for both the locked $(p=0.007)$ and auto modes $(p=0.001)$, with no significant differences between the locked and auto modes $(p>0.99)$ (Figure 8). Individual data indicated that five of the nine subjects had the highest oxygen cost in the locked mode followed by the auto mode and finally 
JRRD, Volume 44, Number 4, 2007

Table.

Data (mean \pm standard deviation) from speed-matched walking trials for nondisabled subjects walking with knee-ankle-foot orthosis that incorporated Stance-Control Orthotic Knee Joint in unlocked, locked, and auto modes.

\begin{tabular}{|c|c|c|c|}
\hline Speed-Matched Data & Unlocked & Locked & Auto \\
\hline Cadence (steps/min) & $90.3 \pm 7.5$ & $89.2 \pm 8.2$ & $91.2 \pm 7.8$ \\
\hline Step Width $(\mathrm{cm})^{*}$ & $14.1 \pm 1.6$ & $17.3 \pm 1.8$ & $15.3 \pm 1.9$ \\
\hline Orthotic-Side Step Length (cm) & $62.5 \pm 9.1$ & $63.4 \pm 6.1$ & $63.1 \pm 6.9$ \\
\hline Nonorthotic-Side Step Length (cm) & $59.4 \pm 10.0$ & $61.3 \pm 7.2$ & $59.8 \pm 9.1$ \\
\hline Orthotic-Side Support Time (gait cycle $\%)^{\dagger}$ & $62.6 \pm 3.1$ & $59.5 \pm 1.9$ & $63.0 \pm 2.7$ \\
\hline Nonorthotic-Side Support Time (gait cycle \%) & $65.5 \pm 2.9$ & $66.4 \pm 2.2$ & $66.7 \pm 1.7$ \\
\hline
\end{tabular}

*Significant difference between unlocked and locked modes, locked and auto modes, and unlocked and auto modes.

${ }^{\dagger}$ Significant difference between unlocked and locked modes.

(a)

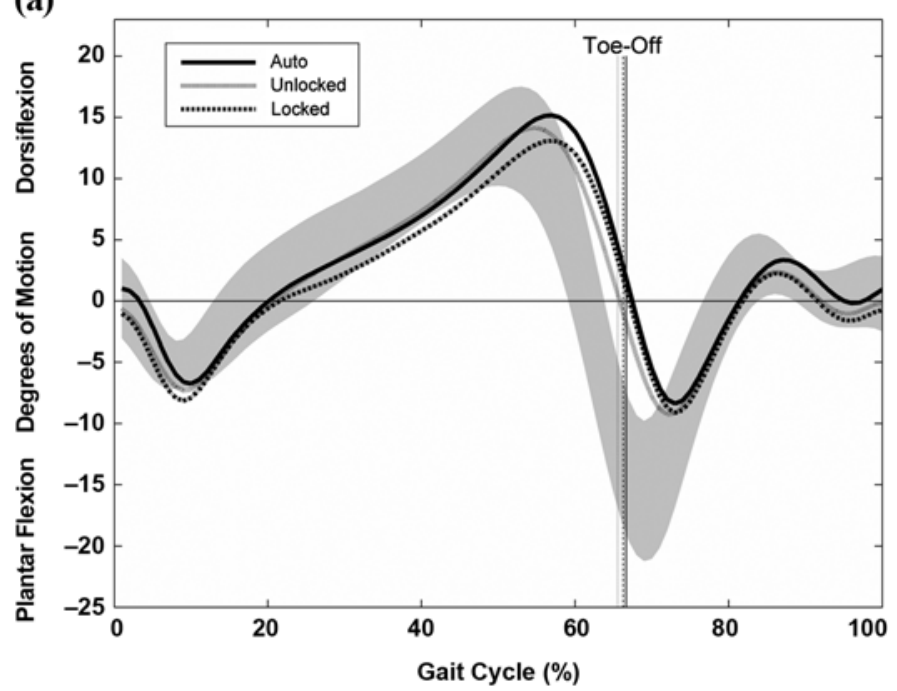

(b)

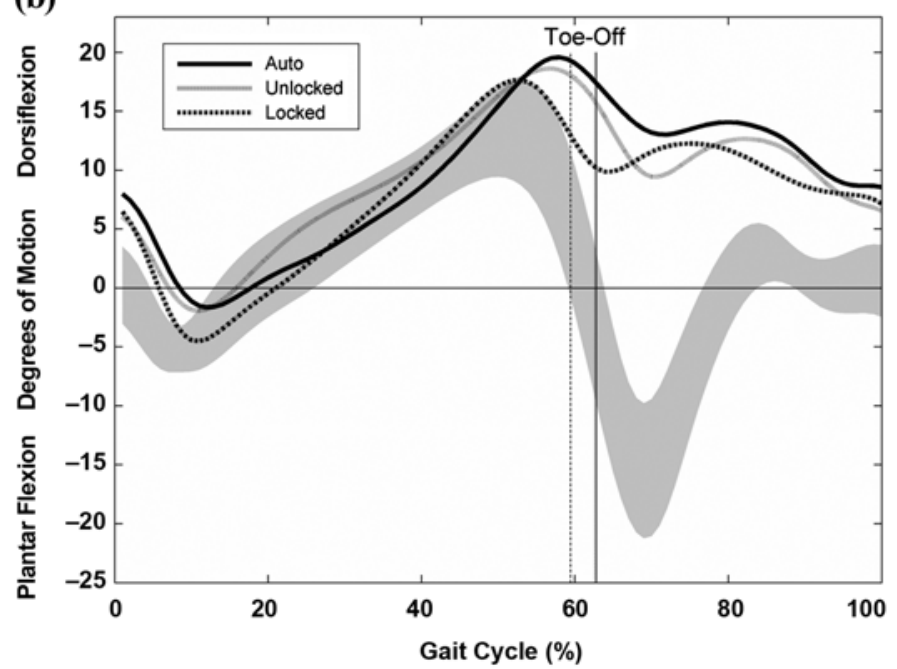

Figure 3.

Average (a) nonorthotic- and (b) orthotic-side ankle dorsiflexion and plantar flexion from speed-matched walking trials for all three modes. Shaded region represents 1 standard deviation about mean for nondisabled subjects walking without orthoses.
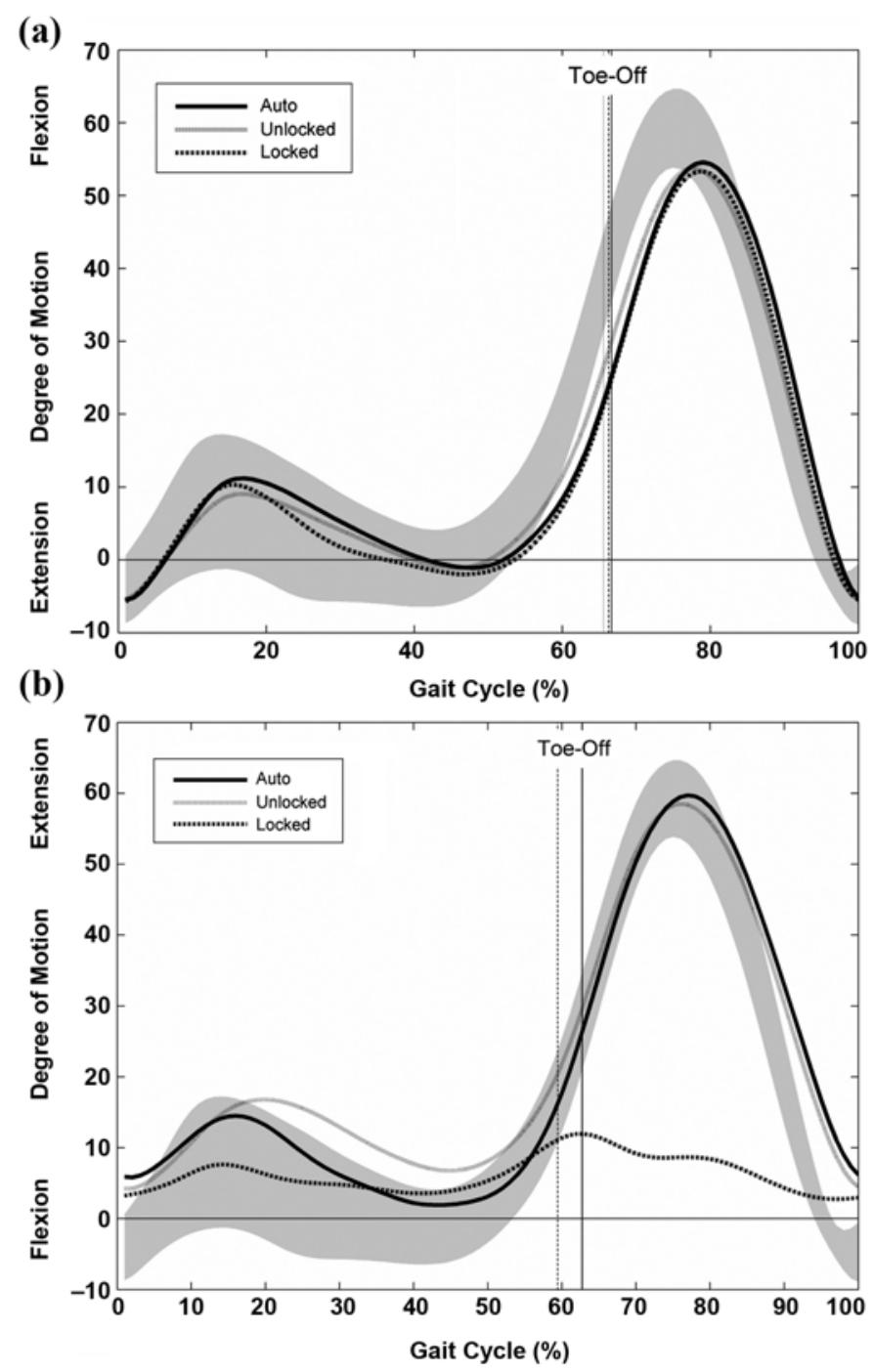

Figure 4.

Average (a) nonorthotic- and (b) orthotic-side knee flexion/extension motion from speed-matched walking trials for all three modes. Shaded region represents 1 standard deviation about mean for nondisabled subjects walking without orthoses. 


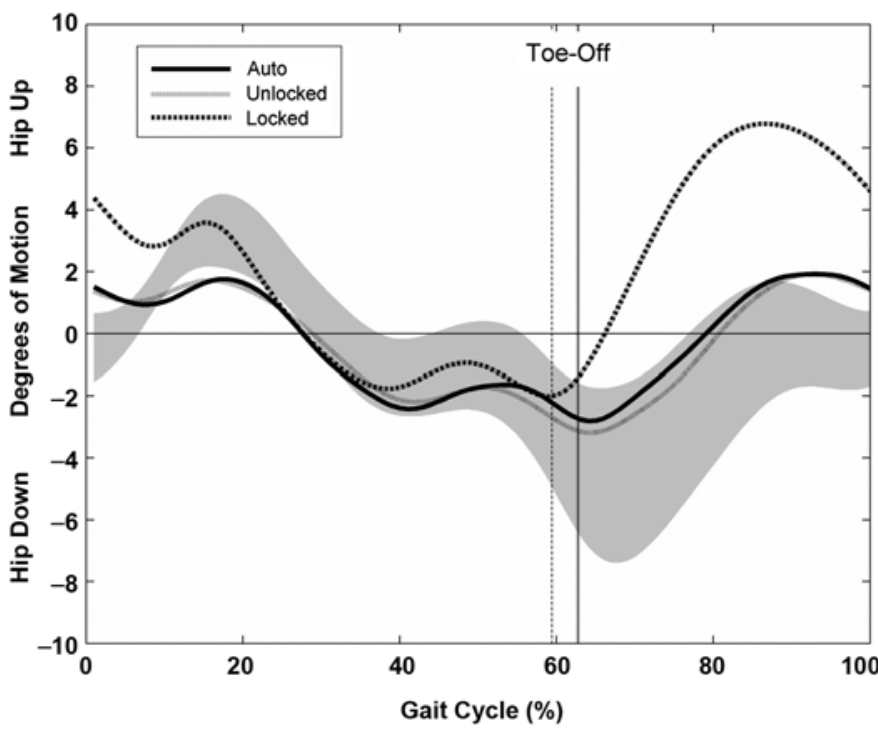

Figure 5.

Average orthotic-side pelvic obliquity from speed-matched walking trials for all three modes. Shaded region represents 1 standard deviation about mean for nondisabled subjects walking without orthoses.

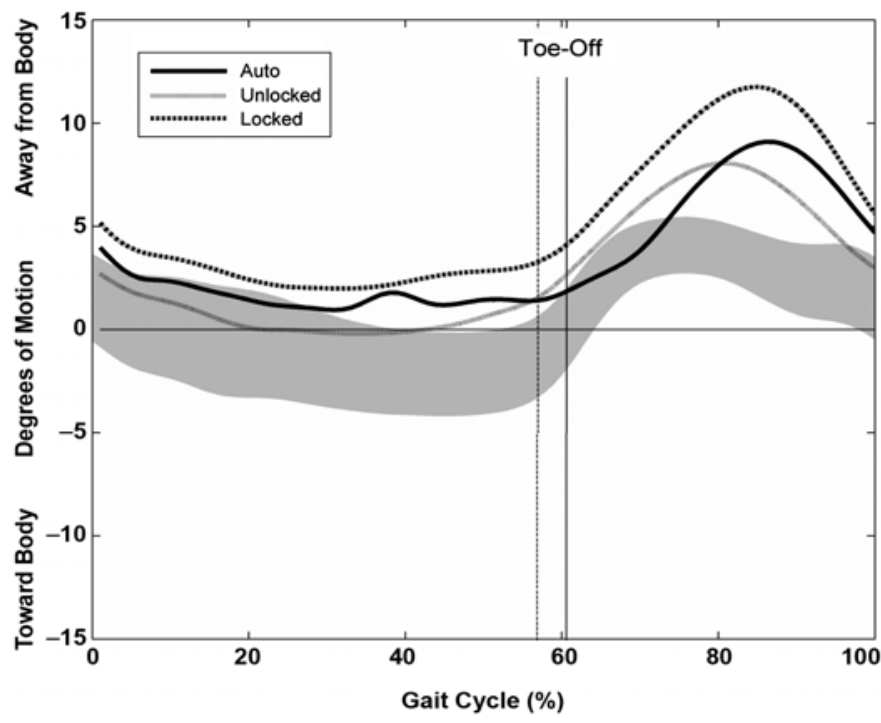

Figure 6.

Speed-matched orthotic-side projection of thigh motion in coronal plane for subject 9 for all three modes. Shaded region represents 1 standard deviation about mean for nondisabled subjects walking without orthoses.

the unlocked mode. For the other four subjects, the auto mode resulted in the highest oxygen cost followed by the locked mode and then the unlocked mode.

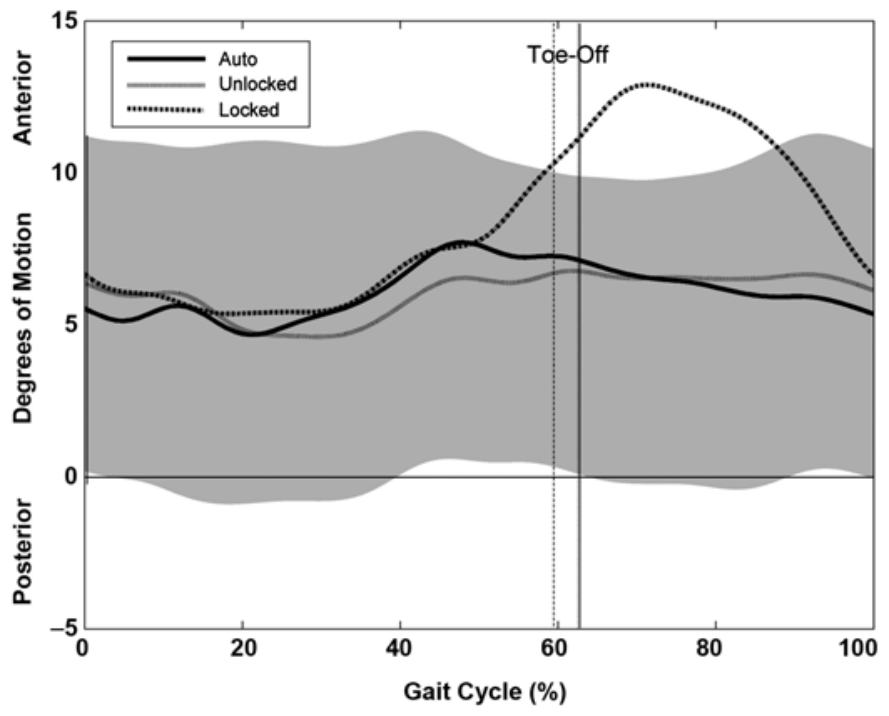

Figure 7.

Average orthotic-side pelvic tilt from speed-matched walking trials for all three modes. Shaded region represents 1 standard deviation about mean for nondisabled subjects walking without orthoses.

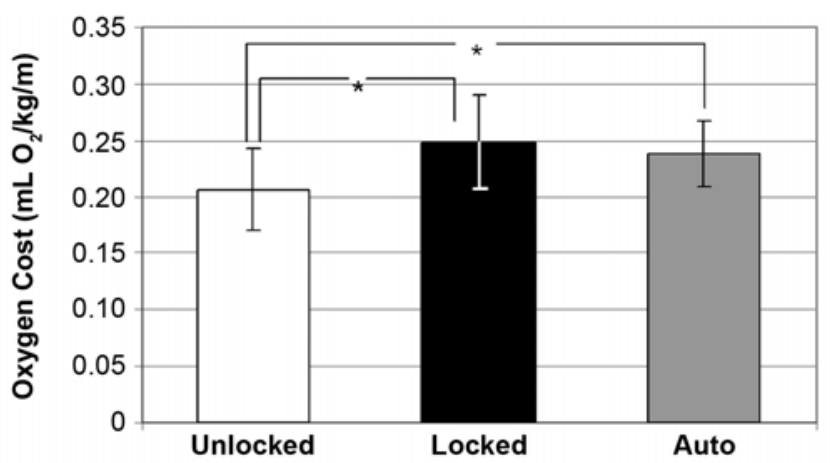

Figure 8.

Average speed-matched oxygen cost shown with 1 standard deviation about mean. * indicates statistically significant differences.

\section{DISCUSSION}

This study investigated the biomechanical and energetic effects of using a KAFO that incorporated the Horton SCOKJ on gait in nondisabled subjects. Data were collected with the SCOKJ in the locked, unlocked, and auto modes for both freely selected and speed-matched conditions. Using nondisabled young adults with normal lower-limb muscle strength and joint range of motion, we were able to analyze the kinematic and energetic effects 
of the SCOKJ on gait in a homogenous population rather than the heterogeneous population typical of KAFO users [11]. This homogeneity allowed us to identify the fundamental effects of orthotic knee joint control on gait. However, nondisabled subjects have greater muscle function and strength and greater joint range of motion than a typical KAFO user probably would have. This may have influenced a number of aspects of the study, including control of the ankle joint, walking speed, and step length variability. For example, during dynamic adjustment of the orthoses, we observed that for the stance-control knee joints to reliably engage and disengage with ankle activation, resistance to plantar flexion had to be increased to such an extent that the ankle joint was held in a dorsiflexed position when at rest and for most of the gait cycle (as shown in Figure 3). It is unlikely that this magnitude of resistance to plantar flexion would be required by persons with gait pathology since they are likely to have less muscle strength than nondisabled subjects. Additionally, orthosis wear time for the nondisabled subjects, which is likely to have been less than that for KAFO users, may have affected walking performance.

In general, the locked mode affected gait the most. During swing phase, the locked mode produced a functionally longer limb by preventing the knee from flexing. All subjects compensated for the loss of knee flexion during swing phase through hip hiking (increased pelvic obliquity), and three subjects additionally used circumduction (increased orthotic-side coronal plane thigh angle during swing phase) to create adequate swing-phase toe clearance. In the auto mode, hip hiking was significantly reduced for the entire group and circumduction was reduced for the three subjects who used this compensation. These results are contrary to those of Hebert and Liggins, who reported that for their single postpolio subject (using an ankle-activated SCOKJ) pelvic obliquity remained close to normal in both modes [10]. However, the current findings are similar to those of McMillan et al., who reported that for their three subjects with gait pathology who were fitted with a weight-activated SCOKJ, the need for compensatory mechanisms was eliminated or largely reduced when the knee was allowed to flex in the swing phase [13]. When making these comparisons, we must consider the findings from the study by Irby et al. [11]. Experienced KAFO users with deeply ingrained walking habits may require a longer accommodation period to adapt to the new gait strategies needed to operate a stance-control device.
Subjects exhibited significantly increased PP pelvic tilt during swing phase in the locked mode. In this mode, the knee joint on the orthotic side was unable to begin flexing in late stance phase. The combination of initial knee flexion and ankle plantar flexion in late stance phase increased the hip-to-toe distance [1], allowing the foot to remain in contact with the ground as weight was transferred to the contralateral limb and providing stability. In the absence of knee flexion during late stance phase, both increased hip extension and ankle plantar flexion could have been used to allow the foot to remain in contact with the ground until weight was transferred to the contralateral limb and swing phase initiated. However, in the locked mode, the hip was already more extended in late stance phase and the double-action orthotic ankle joints (biased toward dorsiflexion) limited the amount of plantar flexion subjects could achieve. Therefore, the pelvis probably tilted anteriorly in the locked mode to allow the trunk to continue its forward progression while the foot remained in contact with the ground.

One unexpected result was that the auto- and lockedmode freely selected walking speeds were not significantly different $(p>0.99)$. These results are consistent with those of Hebert and Liggins, who found temporospatial parameters unchanged between the locked and auto modes for their single postpolio subject [10], but contrary to results reported by McMillan et al., who indicated that speed and cadence were increased in the auto mode compared with the locked mode in their three subjects with gait pathology [13]. In nondisabled subjects, a slower auto-mode walking speed may indicate that the training period provided did not allow the subjects sufficient time to become comfortable and proficient with the operation of the stance-control device. Since it was impractical for nondisabled subjects to wear the SCOKJ all day for consecutive days, training with the device was provided for 20-to-30 minutes each day for up to 10 days. Perhaps a different training strategy was needed to allow subjects to become more proficient at creating a smooth knee-extension motion at the most optimal point in the gait cycle and hence produce faster walking speeds. Alternative training strategies may lead to faster auto-mode walking speeds and should be investigated further since normal walking speed is important for independent community ambulation.

The sensitivity of the push rod (cable) motion to step length was probably most responsible for the slow automode walking speed demonstrated in this study. With the 
ankle-activated SCOKJ, ankle plantar flexion and dorsiflexion create push rod motion, which in turn engage and, along with a small knee-extension movement in late stance phase, disengage the knee joints. During dynamic adjustment, subjects were asked to walk in the auto mode at their comfortable freely selected walking speed. The push rod length and double-action ankle joint were then adjusted until the knee joint properly engaged and disengaged with each step. Since this dynamic adjustment occurred at the beginning of the training period, subjects may have walked slower than their actual comfortable walking speed because of a lack of familiarity with the orthosis.

Another possible explanation for the reduced walking speed may be related to step length and cadence. Changes in step length alter ankle range of motion and therefore push rod motion. The SCOKJ is very sensitive to changes in push rod motion since, according to the manufacturer, only $3 \mathrm{~mm}$ of push rod movement is required to engage and disengage the knee joint. Therefore, subjects in this study seemed to be constrained to the step length and walking speed they used during the initial dynamic adjustment of the push rod. During the training period, we observed that the SCOKJ operated more reliably when subjects walked on the treadmill, where speed was controlled and step length was more uniform, than when they walked overground. It appeared that when subjects altered their speed (and therefore their step length) during over-ground walking, the SCOKJ failed to operate reliably. During the gait evaluation, we also observed that when the SCOKJ failed to operate for one walking trial, subjects were able to adjust their gait to regain proper functioning of the SCOKJ for the next walking trial without manual alteration of the push rod length. This adjustment was achieved by instructing the subject to alter their speed and step length until the joint again functioned correctly. The slow auto-mode walking speed, therefore, was likely because of the mechanics of the device, the setup, and the training provided.

Step length variability and its effect on reliable operation of the ankle-activated SCOKJ may be less of a problem when the device is used by subjects with gait pathology. Persons with muscle weakness and/or joint instability who require a KAFO for ambulation may not be able to alter their step length or walking speed to the extent that a nondisabled person can. Operation of the SCOKJ may therefore be more reliable in these circumstances. Furthermore, this issue can be avoided entirely by using a different activation strategy, such as weight activation, to engage and disengage the knee joints.

While subjects achieved significantly less stancephase knee flexion in the auto mode compared with the unlocked mode ( $p<0.001)$, they exhibited more stancephase knee flexion in the auto mode compared with the locked mode $(p=0.009)$. Since stance-phase knee flexion was designed to be blocked in the auto mode, the auto and locked modes were expected to produce similar amounts of flexion. However, in the auto mode, the cam mechanism had some play that permitted a few degrees of flexion before engaging and blocking knee flexion. This was necessary because a small amount of knee extension was required to disengage the SCOKJ at the end of stance phase. If the knee joints were completely locked in the auto mode (preventing both flexion and extension as in the locked mode), subjects would be unable to create the small extension movement required to push the cam out of the way and disengage the joint. Therefore, play is necessary in the knee joint as reflected by the significantly greater stance-phase knee flexion observed in the auto mode compared with the locked mode. The unlocked mode had no sagittal plane knee-motion constraints and, as expected, the unlocked mode produced significantly higher average stance-phase knee flexion than both the auto and locked modes. Although the auto mode may allow a small amount of stance-phase knee flexion, such flexion may not occur at all when the SCOKJ is used by persons with gait pathology. These users may adopt strategies, such as an anterior trunk lean during stance phase, increased hip extension at initial contact, or application of a posterior force to the thigh with the hand, to ensure that their knees remain fully extended during stance phase. Alternatively, users may develop trust in the device with training and learn to allow this motion. Regardless of the type of user, we currently do not know whether the stance-phase knee flexion achieved in the auto mode is substantial enough to provide functional benefits such as shock absorption [17-18].

Our results indicated that oxygen cost data were not consistent across all subjects; for some subjects, the highest oxygen cost occurred in the locked mode, while for others the highest was in the auto mode. Such inconsistency has also been demonstrated in SCOKJ users with gait pathology. Hebert and Liggins reported a lower PCI in the auto mode compared with the locked mode for their single postpolio subject [10]. McMillan et al. reported a lower heart rate for two of the three subjects in 
their study for the auto mode compared with the locked mode, while the third subject had a higher heart rate for the auto mode compared with the locked mode [13]. In this study, we hypothesized that the auto-mode oxygen cost would be significantly lower than the locked mode, but despite significant reduction in compensatory mechanisms such as hip hiking and circumduction, no significant difference was noted between auto- and lockedmode oxygen cost. The unlocked-mode oxygen cost, on the other hand, was significantly and consistently lower than either of the other two modes $(p<0.007)$. Perhaps the gait strategy needed to operate the device in the auto mode requires a longer training period than what was allowed in this study. With time, subjects could become more accustomed to using the SCOKJ and more habituated to the extension motion at the end of stance phase, decreasing auto-mode energy expenditure. Such a hypothesis could be examined by designing a longitudinal study to track changes in energy expenditure over time and determine the optimal length of gait training. Further studies are required to explore the type of walking (i.e., treadmill, level over ground, or community) and length of gait training that would be most effective for reducing energy expenditure and gait deviations. A noticeable decrease in energy expenditure when walking in the auto mode compared with the locked mode may not exist at all. The gait strategy (and hence muscle activation) needed to operate the SCOKJ in the auto mode may require just as much energy as the compensatory mechanisms adopted for walking in the locked mode. Further research is required to explore this idea.

Overall, the benefits of improved walking kinematics and increased stability during stance phase may be more important than potential reductions in energy expenditure. In the long term, walking with a locked knee may cause quadriceps weakness on the affected side, especially where some muscle strength was initially present. In addition, the need for increased toe clearance when walking with a locked knee can increase the risk of stumbling and make situations such as navigating stairs more difficult. Future research should explore more fully the effects of stance-control orthoses on persons with gait pathology.

\section{CONCLUSIONS}

This study investigated the biomechanical and energetic effects of a KAFO that incorporated the Horton
SCOKJ on gait in nondisabled subjects. Using nondisabled adults with normal lower-limb muscle strength and range of motion allowed us to analyze the kinematic and energetic effects of stance-control orthoses on gait in a homogenous population. Few significant differences were observed in the temporospatial and kinematic data between the auto and unlocked modes. In general, the kinematics for the auto and unlocked modes were more similar than for the auto and locked modes, which indicates that the SCOKJ allowed subjects to walk with a more normal gait pattern. Contrary to our expectations, gait in the auto mode did not yield lower oxygen cost compared with the locked mode. A longer training period than that provided in this study may be necessary for subjects to fully adapt to operating the SCOKJ in the auto mode. Additionally, the type of gait training could be examined to determine how to most effectively teach users to properly operate the SCOKJ. Further studies are required to establish the effects of stance-control orthoses on persons with gait pathology.

\section{ACKNOWLEDGMENTS}

Data for this project were collected at the VACMARL, Jesse Brown VA Medical Center, Chicago, Illinois.

This material was based on work supported by the National Institute on Disability and Rehabilitation Research of the U.S. Department of Education under grant H133E030030.

The opinions contained in this article are those of the grantee and do not necessarily reflect those of the U.S. Department of Education.

The authors have declared that no competing interests exist.

\section{REFERENCES}

1. Gard SA, Fatone S. Biomechanics of lower limb function and gait. In: Condie E, Campbell J, Martina J, editors. Report of a consensus conference on the orthotic management of stroke patients. Copenhagen (Denmark): International Society for Prosthetics and Orthotics; 2003. p. 55-63.

2. Kerrigan DC, Frates EP, Rogan S, Riley PO. Hip hiking and circumduction: Quantitative definitions. Am J Phys Med Rehabil. 2000;79(3):247-52. [PMID: 10821310$]$ 
3. Lage KJ, White SC, Yack HJ. The effects of unilateral knee immobilization on lower extremity gait mechanics. Med Sci Sports Exerc. 1995;27(1):8-14. [PMID: 7898343]

4. Mattsson E, Broström LA. The increase in energy cost of walking with an immobilized knee or an unstable ankle. Scand J Rehabil Med. 1990;22(1):51-53. [PMID: 2326610]

5. Ralston HJ. Effects of immobilization of various body segments on the energy cost of human locomotion. Ergonomics. 1965;Suppl:53-60.

6. Waters RL, Campbell J, Thomas L, Hugos L, Davis P. Energy costs of walking in lower-extremity plaster casts. J Bone Joint Surg Am. 1982;64(6):896-99. [PMID: 7085717]

7. Hanada E, Kerrigan DC. Energy consumption during level walking with arm and knee immobilized. Arch Phys Med Rehabil. 2001;82(9):1251-54. [PMID: 11552199]

8. Kaufman KR, Irby SE, Mathewson JW, Wirta RW, Sutherland DH. Energy-efficient knee-ankle-foot orthosis. J Prosthet Orthot. 1996;8(3):79-85.

9. Fatone S. A review of the literature pertaining to KAFOs and HKAFOs for ambulation. J Prosthet Orthot. 2006; 18(3S):137-68.

10. Hebert JS, Liggins AB. Gait evaluation of an automatic stance-control knee orthosis in a patient with postpoliomyelitis. Arch Phys Med Rehabil. 2005;86(8):1676-80. [PMID: 16084826]

11. Irby SE, Bernhardt KA, Kaufman KR. Gait of stance control orthosis users: The dynamic knee brace system. Prosthet Orthot Int. 2005;29(3):269-82. [PMID: 16466156]
12. Lehmann JF, Stonebridge JB. Knee lock device for knee ankle orthoses for spinal cord injured patients: An evaluation. Arch Phys Med Rehabil. 1978;59(5):207-11. [PMID: 655831]

13. McMillan AG, Kendrick K, Michael JW, Aronson J, Horton GW. Preliminary evidence for effectiveness of a stance control orthosis. J Prosthet Orthot. 2004;16(1):6-13.

14. Suga T, Kameyama O, Ogawa R, Matsuura M, Oka H. Newly designed computer controlled knee-ankle-foot orthosis (Intelligent Orthosis). Prosthet Orthot Int. 1998;22(3): 230-39. [PMID: 9881611]

15. Kadaba MP, Ramakrishnan HK, Wootten ME. Measurement of lower extremity kinematics during level walking. J Orthop Res. 1990;8(3):383-92. [PMID: 2324857]

16. Field A. Discovering statistics using SPSS for Windows. Thousand Oaks (CA): SAGE Publications; 2000.

17. Gard SA, Childress DS. The influence of stance-phase knee flexion on the vertical displacement of the trunk during normal walking. Arch Phys Med Rehabil. 1999;80(1): 26-32. [PMID: 9915368]

18. Perry J. Gait analysis: Normal and pathological function. Thorofare (NJ): SLACK Incorporated; 1992.

Submitted for publication September 29, 2006. Accepted in revised form February 27, 2007. 